\title{
ASYMPTOTIC FORMULAS AND CRITICAL EXPONENTS FOR TWO-PARAMETER NONLINEAR EIGENVALUE PROBLEMS
}

TETSUTARO SHIBATA

Received 17 January 2002

We study the nonlinear two-parameter problem $-u^{\prime \prime}(x)+\lambda u(x)^{q}=\mu u(x)^{p}$, $u(x)>0, x \in(0,1), u(0)=u(1)=0$. Here, $1<q<p$ are constants and $\lambda, \mu>0$ are parameters. We establish precise asymptotic formulas with exact second term for variational eigencurve $\mu(\lambda)$ as $\lambda \rightarrow \infty$. We emphasize that the critical case concerning the decaying rate of the second term is $p=(3 q-1) / 2$ and this kind of criticality is new for two-parameter problems.

\section{Introduction}

We consider the following nonlinear two-parameter problem:

$$
\begin{gathered}
-u^{\prime \prime}(x)+\lambda u(x)^{q}=\mu u(x)^{p}, \quad x \in I=(0,1), \\
u(x)>0, \quad x \in I, \\
u(0)=u(1)=0,
\end{gathered}
$$

where $1<q<p$ and $\lambda, \mu>0$ are parameters.

The purpose of this paper is to establish the asymptotic formulas for the eigencurve $\mu=\mu(\lambda)$ with the exact second term as $\lambda \rightarrow \infty$ by using a variational method. We also establish the critical relationship between $p$ and $q$ from a viewpoint of the decaying rate of the second term of $\mu(\lambda)$.

The study of two-parameter eigenvalue problems began with the oscillation theory and has been investigated by many authors. We refer to $[1,2,3,4,5$, $6,7,8,9,10,11]$ and the references therein. One of the main problems in this area is to analyze the structure of the solution set $\{(\lambda, \mu, u)\}$ of $(1.1)$, and the effective approach to this problem is to study the structure of the set $S_{\lambda, \mu}:=$ $\left\{\left(\lambda, \mu,\|u\|_{p+1}\right)\right\} \subset \mathbb{R}^{3}$ for large $\lambda$. In Shibata [7], by using a standard variational framework (see Section 2), the variational eigencurve $\mu=\mu(\lambda)$ was defined to 
analyze $S_{\lambda, \mu}$ and the following asymptotic formula for $\mu(\lambda)$ as $\lambda \rightarrow \infty$ was established:

$$
\mu(\lambda)=C_{1} \lambda^{(p+3) /(2 p-q+3)}+o\left(\lambda^{(p+3) /(2 p-q+3)}\right),
$$

where

$$
\begin{gathered}
C_{1}=\left(\frac{(p+1)(q+3)}{(p+3)(q+1)} \frac{1}{\gamma^{p+1}} \frac{2}{p-q} \sqrt{\frac{\pi(q+1)}{2}}\left(\frac{p+1}{q+1}\right)^{(q+3) / 2(p-q)}\right. \\
\left.\times \frac{\Gamma((q+3) / 2(p-q))}{\Gamma((p+3) / 2(p-q))}\right)^{2(p-q) /(2 p-q+3)} \\
\Gamma(r)=\int_{0}^{\infty} y^{r-1} e^{-y} d y \quad(r>0) .
\end{gathered}
$$

By this formula, we understood the first term of $\mu(\lambda)$ as $\lambda \rightarrow \infty$. However, the remainder estimate of $\mu(\lambda)$ has not been obtained. The purpose here is to obtain the exact second term of $\mu(\lambda)$ as $\lambda \rightarrow \infty$. We emphasize that the second term depends deeply on the relationship between $p$ and $q$, and the critical case is $p=(3 q-1) / 2$. More precisely, if $p=(3 q-1) / 2$, then the asymptotic behavior of the second term of $\mu(\lambda)$ is completely different from that of the case where $p \neq(3 q-1) / 2$. As far as we know, this kind of criticality is new for twoparameter problems and great interest by itself. Finally, it should be mentioned that the asymptotic behavior of such eigencurve is also effected by the variational framework (cf. $[6,8])$.

\section{Main results}

We explain notations before stating our results. Let $H_{0}^{1}(I)$ be the usual real Sobolev space. Let $\|u\|_{r}$ denote the usual $L^{r}$-norm. For $u \in H_{0}^{1}(I)$,

$$
\begin{aligned}
E_{\lambda}(u) & :=\frac{1}{2}\left\|u^{\prime}\right\|_{2}^{2}+\frac{1}{q+1} \lambda\|u\|_{q+1}^{q+1}, \\
M_{\gamma} & :=\left\{u \in H_{0}^{1}(I):\|u\|_{p+1}=\gamma\right\},
\end{aligned}
$$

where $\gamma>0$ is a fixed constant. For a given $\lambda>0$, we call $\mu(\lambda)$ the variational eigenvalue when the following conditions are satisfied:

$$
\begin{gathered}
\left(\lambda, \mu(\lambda), u_{\lambda}\right) \in \mathbb{R}_{+} \times \mathbb{R}_{+} \times M_{\gamma} \quad \text { satisfies (1.1), } \\
E_{\lambda}\left(u_{\lambda}\right)=\inf _{u \in M_{\gamma}} E_{\lambda}(u) .
\end{gathered}
$$

Then $\mu(\lambda)$ is obtained as a Lagrange multiplier and is represented explicitly as follows:

$$
\mu(\lambda)=\frac{\left\|u_{\lambda}^{\prime}\right\|_{2}^{2}+\lambda\left\|u_{\lambda}\right\|_{q+1}^{q+1}}{\gamma^{p+1}}
$$


Indeed, multiply the equation in (1.1) by $\mathcal{u}_{\lambda}$. Then integration by parts yields

$$
\left\|u_{\lambda}^{\prime}\right\|_{2}^{2}+\lambda\left\|u_{\lambda}\right\|_{q+1}^{q+1}=\mu(\lambda)\left\|u_{\lambda}\right\|_{p+1}^{p+1}=\mu(\lambda) \gamma^{p+1}
$$

This implies (2.3). The existence of $\mu(\lambda)$ for a given $\lambda>0$ is ensured in [7, Theorem 2.1] and $\mu(\lambda)$ is continuous for $\lambda>0$ (cf. [7, Theorem 2.2]). Finally, let

$$
\begin{aligned}
K_{1}:= & \left(\sqrt{2}\left(\frac{q+1}{p+1}\right)^{(q-1) /(2(p-q))} \frac{\Gamma(1 /(q+1)) \Gamma((q-1) / 2(q+1))}{\sqrt{\pi(q+1)}}\right. \\
& \left.\quad \times C_{1}^{(q-1) /(2(p-q))}\right)^{2(q+1) /(q-1)}, \\
K_{2}:= & \frac{1}{2} \int_{0}^{1} \frac{s^{(2 p-3 q-1) / 2}\left(1-s^{p+1}\right)}{\left(1-s^{p-q}\right)^{3 / 2}} d s, \\
K_{3}:= & \frac{2^{2(p+2) /(q+1)}}{q+1} \int_{0}^{1} \frac{y^{(2 p-2 q+2) /(q+1)}}{(1+y)^{2(p+2) /(q+1)}(1-y)^{(2 p-2 q+2) /(q+1)}} d y, \\
J_{0}= & \frac{\sqrt{\pi}}{p-q} \frac{q+3}{p+3} \frac{\Gamma((q+3) / 2(p-q))}{\Gamma((p+3) / 2(p-q))} .
\end{aligned}
$$

Now, we state our results.

Theorem 2.1. (1) Assume $p>(3 q-1) / 2$. Then the following asymptotic formula holds as $\lambda \rightarrow \infty$ :

$$
\mu(\lambda)=C_{1} \lambda^{(p+3) /(2 p-q+3)}\left\{1+C_{2}(1+o(1)) \lambda^{-2(p+1)(q+1) /((2 p-q+3)(q-1))}\right\},
$$

where

$$
C_{2}=K_{1}\left(1-\frac{2(p-q) K_{2}}{(2 p-q+3) J_{0}}\right)
$$

(2) Assume $p<(3 q-1) / 2$. Then as $\lambda \rightarrow \infty$,

$$
\mu(\lambda)=C_{1} \lambda^{(p+3) /(2 p-q+3)}\left\{1-C_{3}(1+o(1)) \lambda^{-(p+1) /(q-1)}\right\},
$$

where

$$
C_{3}=\frac{2(p-q)}{(2 p-q+3) J_{0}} K_{3} K_{1}^{(2 p-q+3) /(2(q+1))}
$$

(3) Assume $p=(3 q-1) / 2$. Then as $\lambda \rightarrow \infty$,

$$
\mu(\lambda)=C_{1} \lambda^{(p+3) /(2 p-q+3)}\left\{1-C_{4}(1+o(1)) \lambda^{-2(p+1)(q+1) /((2 p-q+3)(q-1))} \log \lambda\right\},
$$


674 Two-parameter eigenvalue problems

where

$$
C_{4}=\frac{2(p-q)(p+1)}{(q-1)(2 p-q+3)^{2} J_{0}} K_{1}
$$

We briefly explain the idea of the proof. Put

$$
\begin{aligned}
v(\lambda) & =\lambda^{(p-1) / 2(p-q)} \mu(\lambda)^{(1-q) / 2(p-q)} \\
w_{\lambda}(t) & =\left(\frac{\mu(\lambda)}{\lambda}\right)^{1 /(p-q)} u_{\lambda}(x), \quad t=\nu(\lambda)\left(x-\frac{1}{2}\right) .
\end{aligned}
$$

Then it follows from (1.1) that $w_{\lambda}$ satisfies

$$
\begin{gathered}
-w_{\lambda}^{\prime \prime}(t)=w_{\lambda}(t)^{p}-w_{\lambda}(t)^{q}, \quad t \in I_{\nu(\lambda)}:=\left(-\frac{1}{2} \nu(\lambda), \frac{1}{2} \nu(\lambda)\right), \\
w_{\lambda}(t)>0, \quad t \in I_{\nu(\lambda)}, \\
w_{\lambda}\left( \pm \frac{1}{2} \nu(\lambda)\right)=0 .
\end{gathered}
$$

Then by [7, Lemma 5.1],

$$
\nu(\lambda) \longrightarrow \infty
$$

as $\lambda \rightarrow \infty$. Put $z_{\lambda}=w_{\lambda} /\left\|w_{\lambda}\right\|_{\infty}$. Then it is easy to see from (2.3) that

$$
\begin{aligned}
\mu(\lambda) & =\frac{\lambda^{(p+3) /(2(p-q))} \mu(\lambda)^{-(q+3) /(2(p-q))}\left(\left\|w_{\lambda}^{\prime}\right\|_{2}^{2}+\left\|w_{\lambda}\right\|_{q+1}^{q+1}\right)}{\gamma^{p+1}} \\
& =\frac{\lambda^{(p+3) /(2(p-q))} \mu(\lambda)^{-(q+3) /(2(p-q))}\left\|w_{\lambda}\right\|_{p+1}^{p+1}}{\gamma^{p+1}} \\
& =\frac{\lambda^{(p+3) /(2(p-q))} \mu(\lambda)^{-(q+3) /(2(p-q))}\left\|w_{\lambda}\right\|_{\infty}^{p+1}\left\|z_{\lambda}\right\|_{p+1}^{p+1}}{\gamma^{p+1}} .
\end{aligned}
$$

Therefore, it is crucial to study the asymptotic behavior of $\left\|w_{\lambda}\right\|_{\infty}$ and $\left\|z_{\lambda}\right\|_{p+1}$ as $\lambda \rightarrow \infty$.

\section{Asymptotic behavior of $\left\|w_{\lambda}\right\|_{\infty}$}

In this section, we study the asymptotic behavior of $\left\|w_{\lambda}\right\|_{\infty}$ as $\lambda \rightarrow \infty$. We put

$$
\left\|w_{\lambda}\right\|_{\infty}=\left(\frac{p+1}{q+1}(1+\epsilon(\lambda))\right)^{1 /(p-q)} .
$$

Then by [7, (5.10), Lemma 5.2], we know that $\epsilon(\lambda)>0$ and $\epsilon(\lambda) \rightarrow 0$ as $\lambda \rightarrow \infty$. 
Lemma 3.1. The following equality holds for $\lambda>0$ :

$$
\nu(\lambda)=\sqrt{2(q+1)}\left(\frac{p+1}{q+1}(1+\epsilon(\lambda))\right)^{-(q-1) /(2(p-q))} L(\epsilon(\lambda)),
$$

where

$$
\begin{gathered}
L(\epsilon)=\int_{0}^{1} \frac{1}{m(\epsilon, s)} d s, \\
m(\epsilon, s)=\sqrt{s^{q+1}-s^{p+1}+\epsilon\left(1-s^{p+1}\right)} \quad(\epsilon>0) .
\end{gathered}
$$

Proof. Multiply the equation in (2.13) by $w_{\lambda}^{\prime}$. Then for $t \in I_{\nu(\lambda)}$,

$$
w_{\lambda}^{\prime \prime}(t) w_{\lambda}^{\prime}(t)+w_{\lambda}(t)^{p} w_{\lambda}^{\prime}(t)-w_{\lambda}(t)^{q} w_{\lambda}^{\prime}(t)=0,
$$

which implies that

$$
\frac{d}{d t}\left(\frac{1}{2}\left(w_{\lambda}^{\prime}(t)\right)^{2}+\frac{1}{p+1} w_{\lambda}(t)^{p+1}-\frac{1}{q+1} w_{\lambda}(t)^{q+1}\right)=0 .
$$

We know that $w_{\lambda}(0)=\left\|w_{\lambda}\right\|_{\infty}$ and $w_{\lambda}^{\prime}(0)=0$ since $u_{\lambda}(1 / 2)=\left\|u_{\lambda}\right\|_{\infty}$ and $u_{\lambda}^{\prime}(1 / 2)=0$. Then put $t=0$ to obtain

$$
\frac{1}{2} w_{\lambda}^{\prime}(t)^{2}+\frac{1}{p+1} w_{\lambda}(t)^{p+1}-\frac{1}{q+1} w_{\lambda}(t)^{q+1} \equiv \frac{1}{p+1}\left\|w_{\lambda}\right\|_{\infty}^{p+1}-\frac{1}{q+1}\left\|w_{\lambda}\right\|_{\infty}^{q+1} .
$$

Note that $w_{\lambda}^{\prime}(t)<0$ for $t \in(0, v(\lambda) / 2)$ since $u_{\lambda}^{\prime}(x)<0$ for $x \in(1 / 2,1)$. Then it follows from this and (3.1) that for $t \in(0, v(\lambda) / 2)$,

$$
\begin{aligned}
-z_{\lambda}^{\prime}(t) & =\left\|w_{\lambda}\right\|_{\infty}^{(q-1) / 2} \sqrt{\frac{2}{q+1}} \sqrt{z_{\lambda}(t)^{q+1}-z_{\lambda}(t)^{p+1}+\epsilon(\lambda)\left(1-z_{\lambda}(t)^{p+1}\right)} \\
& =\left\|w_{\lambda}\right\|_{\infty}^{(q-1) / 2} \sqrt{\frac{2}{q+1}} m\left(\epsilon(\lambda), z_{\lambda}(t)\right) .
\end{aligned}
$$

Put $s=z_{\lambda}$. Then (3.1) and (3.7) yield

$$
\begin{aligned}
\frac{\nu(\lambda)}{2} & =\int_{0}^{\nu(\lambda) / 2} \frac{-z_{\lambda}^{\prime}(t)}{\sqrt{2 /(q+1)}\left\|w_{\lambda}\right\|_{\infty}^{(q-1) / 2} m\left(\epsilon(\lambda), z_{\lambda}(t)\right)} d t \\
& =\sqrt{\frac{q+1}{2}}\left(\frac{p+1}{q+1}(1+\epsilon(\lambda))\right)^{-(q-1) /(2(p-q))} \int_{0}^{1} \frac{1}{m(\epsilon(\lambda), s)} d s .
\end{aligned}
$$

This implies (3.2). 
In order to study the asymptotic behavior of $\epsilon(\lambda)$ as $\lambda \rightarrow \infty$, we investigate the asymptotic behavior of $L(\epsilon)$ as $\epsilon \rightarrow 0$.

Lemma 3.2. For $0<\epsilon \ll 1$,

$$
L(\epsilon)=\frac{\Gamma(1 /(q+1)) \Gamma((q-1) / 2(q+1))}{(q+1) \sqrt{\pi}} \epsilon^{-(q-1) /(2(q+1))}+o\left(\epsilon^{-(q-1) /(2(q+1))}\right)
$$

Proof. Put

$$
L_{1}(\epsilon):=L(\epsilon)-\int_{0}^{1} \frac{1}{\sqrt{s^{q+1}+\epsilon}} d s
$$

Put $s=\epsilon^{1 /(q+1)} \tan ^{2 /(q+1)} \theta$. Then

$$
\begin{aligned}
\int_{0}^{1} \frac{1}{\sqrt{s^{q+1}+\epsilon}} d s \\
=\frac{2}{q+1} \epsilon^{-(q-1) /(2(q+1))} \int_{0}^{\tan ^{-1}(1 / \sqrt{\epsilon})} \sin ^{-(q-1) /(q+1)} \theta \cos ^{-2 /(q+1)} \theta d \theta \\
=\frac{2}{q+1}(1+o(1)) \epsilon^{-(q-1) /(2(q+1))} \int_{0}^{\pi / 2} \sin ^{-(q-1) /(q+1)} \theta \cos ^{-2 /(q+1)} \theta d \theta \\
=\frac{1}{q+1}(1+o(1)) \epsilon^{-(q-1) /(2(q+1))} B\left(\frac{1}{q+1}, \frac{q-1}{2(q+1)}\right) \\
=\frac{1}{q+1}(1+o(1)) \epsilon^{-(q-1) /(2(q+1))} \frac{\Gamma(1 /(q+1)) \Gamma((q-1) / 2(q+1))}{\Gamma(1 / 2)} \\
=\frac{1}{q+1}(1+o(1)) \epsilon^{-(q-1) /(2(q+1))} \frac{\Gamma(1 /(q+1)) \Gamma((q-1) / 2(q+1))}{\sqrt{\pi}} .
\end{aligned}
$$

We use here the formula

$$
2 \int_{0}^{\pi / 2} \sin ^{2 m-1} \theta \cos ^{2 n-1} \theta d \theta=B(m, n)=\frac{\Gamma(m) \Gamma(n)}{\Gamma(m+n)} \quad(m, n>0)
$$

where $B(m, n)$ is the beta function. Next, we calculate $L_{1}(\epsilon)$. Note that for $0 \leq$ $s \leq 1$,

$$
m(\epsilon, s)=\sqrt{s^{q+1}\left(1-s^{p-q}\right)+\epsilon\left(1-s^{p+1}\right)} \geq \sqrt{\left(s^{q+1}+\epsilon\right)\left(1-s^{p-q}\right)} .
$$


By this, we obtain

$$
\begin{aligned}
& \left|L_{1}(\epsilon)\right| \\
& =\int_{0}^{1} \frac{(1+\epsilon) s^{p+1}}{m(\epsilon, s) \sqrt{s^{q+1}+\epsilon}\left(m(\epsilon, s)+\sqrt{s^{q+1}+\epsilon}\right)} d s \\
& \leq \int_{0}^{1} \frac{(1+\epsilon) s^{p+1}}{\sqrt{\left(s^{q+1}+\epsilon\right)\left(1-s^{p-q}\right)} \sqrt{s^{q+1}+\epsilon}\left(\sqrt{\left(s^{q+1}+\epsilon\right)\left(1-s^{p-q}\right)}+\sqrt{s^{q+1}+\epsilon}\right)} d s \\
& \leq(1+\epsilon) \int_{0}^{1} \frac{s^{p+1}}{\left(s^{q+1}+\epsilon\right)^{3 / 2} \sqrt{1-s^{p-q}}\left(1+\sqrt{1-s^{p-q}}\right)} d s \\
& \leq 2 \int_{0}^{1} \frac{s^{p+1}}{\left(s^{q+1}+\epsilon\right)^{3 / 2} \sqrt{1-s^{p-q}}} d s \\
& =2 \int_{0}^{\delta} \frac{s^{p+1}}{\left(s^{q+1}+\epsilon\right)^{3 / 2} \sqrt{1-s^{p-q}}} d s+2 \int_{\delta}^{1} \frac{s^{p+1}}{\left(s^{q+1}+\epsilon\right)^{3 / 2} \sqrt{1-s^{p-q}}} d s \\
& :=I+I I \text {, }
\end{aligned}
$$

where $0<\delta \ll 1$ is a fixed constant. Let $C_{j, \delta}>0(j=1,2, \ldots)$ be constants depending only on $\delta$. Put $s=\sin ^{2 /(p-q)} \theta$. Then

$$
\begin{aligned}
I I & \leq \frac{2}{\delta^{3(q+1) / 2}} \int_{\delta}^{1} \frac{1}{\sqrt{1-s^{p-q}}} d s \\
& =\frac{2}{\delta^{3(q+1) / 2}} \frac{2}{p-q} \int_{\sin ^{-1} \delta^{(p-q) / 2}}^{1} \sin ^{(2+q-p) /(p-q)} \theta d \theta \\
& \leq C_{1, \delta} .
\end{aligned}
$$

Moreover, put $s=\epsilon^{1 /(q+1)} t$. Then for $0<\epsilon \ll 1$,

$$
\begin{aligned}
I & \leq \frac{2}{\sqrt{1-\delta^{p-q}}} \int_{0}^{\delta} \frac{\epsilon^{(p+1) /(q+1)} t^{p+1}}{\epsilon^{3 / 2}\left(t^{q+1}+1\right)^{3 / 2}} \epsilon^{1 /(q+1)} d t \\
& \leq 2 \frac{\delta^{p+1}}{\sqrt{1-\delta^{p-q}}} \epsilon^{(2 p-3 q+1) /(2(q+1))}=o\left(\epsilon^{-(q-1) /(2(q+1))}\right) .
\end{aligned}
$$

By (3.14), (3.15), and (3.16), we have

$$
\left|L_{1}(\epsilon)\right|=o\left(\epsilon^{-(q-1) /(2(q+1))}\right) .
$$

By this, (3.10), and (3.11), we obtain (3.9).

Now, we study the asymptotic behavior of $\epsilon(\lambda)$ as $\lambda \rightarrow \infty$.

Lemma 3.3. As $\lambda \rightarrow \infty$,

$$
\epsilon(\lambda)=K_{1}(1+o(1)) \lambda^{-2(p+1)(q+1) /((q-1)(2 p-q+3))} .
$$


678 Two-parameter eigenvalue problems

Proof. By (1.2) and (2.12), we have

$$
\begin{aligned}
\nu(\lambda) & =\lambda^{(p-1) /(2(p-q))} \mu(\lambda)^{(1-q) /(2(p-q))} \\
& =\lambda^{(p-1) /(2(p-q))}\left(C_{1} \lambda^{(p+3) /(2 p-q+3)}\right)^{(1-q) /(2(p-q))}(1+o(1)) \\
& =C_{1}^{(1-q) /(2(p-q))}(1+o(1)) \lambda^{(p+1) /(2 p-q+3)} .
\end{aligned}
$$

On the other hand, by Lemmas 3.1 and 3.2 and Taylor expansion, we have

$$
\begin{aligned}
\nu(\lambda)= & \sqrt{2(q+1)}\left(\frac{p+1}{q+1}\right)^{-(q-1) /(2(p-q))}(1+\epsilon(\lambda))^{-(q-1) /(2(p-q))} L(\epsilon(\lambda)) \\
= & \sqrt{2(q+1)}\left(\frac{p+1}{q+1}\right)^{-(q-1) /(2(p-q))}\left(1-\frac{q-1}{2(p-q)} \epsilon(\lambda)+o(\epsilon(\lambda))\right) \\
& \times\left(\frac{\Gamma(1 /(q+1)) \Gamma((q-1) / 2(q+1))}{(q+1) \sqrt{\pi}} \epsilon(\lambda)^{-(q-1) /(2(q+1))}\right. \\
& \left.\quad+o\left(\epsilon(\lambda)^{-(q-1) /(2(q+1))}\right)\right) \\
= & \sqrt{2}\left(\frac{p+1}{q+1}\right)^{-(q-1) /(2(p-q))} \frac{\Gamma(1 /(q+1)) \Gamma((q-1) / 2(q+1))}{\sqrt{\pi(q+1)}} \\
& \times \epsilon(\lambda)^{-(q-1) /(2(q+1))}(1+o(1)) .
\end{aligned}
$$

By this and (3.19), we obtain (3.18).

4. Asymptotic behavior of $\left\|z_{\lambda}\right\|_{p+1}$

In this section, we calculate $\left\|z_{\lambda}\right\|_{p+1}$. Note that $z_{\lambda}(t)=z_{\lambda}(-t)$ for $t \in I_{\nu(\lambda)}$. Then by (3.7) and putting $s=z_{\lambda}(t)$, we have

$$
\begin{aligned}
\left\|z_{\lambda}\right\|_{p+1}^{p+1} & =2 \int_{0}^{\nu(\lambda) / 2} z_{\lambda}(t)^{p+1} d t \\
& =2 \int_{0}^{\nu(\lambda) / 2} z_{\lambda}(t)^{p+1} \frac{-z_{\lambda}^{\prime}(t)}{\left\|w_{\lambda}\right\|_{\infty}^{(q-1) / 2} \sqrt{2 /(q+1)} m\left(\epsilon(\lambda), z_{\lambda}(t)\right)} d t \\
& =\frac{\sqrt{2(q+1)}}{\left\|w_{\lambda}\right\|_{\infty}^{(q-1) / 2}} J(\epsilon(\lambda)),
\end{aligned}
$$

where

$$
J(\epsilon):=\int_{0}^{1} \frac{s^{p+1}}{m(\epsilon, s)} d s \quad(\epsilon>0) .
$$


Therefore, we study the precise asymptotics of $J(\epsilon)$ as $\epsilon \rightarrow 0$. Put $s=\sin ^{2 /(p-q)} \theta$. Then as $\epsilon \rightarrow 0$,

$$
\begin{aligned}
J(\epsilon) \longrightarrow J(0) & =\int_{0}^{1} \frac{s^{(2 p-q+1) / 2}}{\sqrt{1-s^{p-q}}} d s \\
& =\frac{2}{p-q} \int_{0}^{\pi / 2} \sin ^{(p+3) /(p-q)} \theta d \theta \\
& =\frac{\sqrt{\pi}}{p-q} \frac{q+3}{p+3} \frac{\Gamma((q+3) / 2(p-q))}{\Gamma((p+3) / 2(p-q))}=J_{0} .
\end{aligned}
$$

We use here the formulas

$$
\begin{gathered}
\int_{0}^{\pi / 2} \sin ^{r} \theta d \theta=\frac{\sqrt{\pi}}{2} \frac{\Gamma((r+1) / 2)}{\Gamma(r / 2+1)} \quad(r>-1), \\
\Gamma(r+1)=r \Gamma(r) .
\end{gathered}
$$

Therefore, put

$$
\begin{gathered}
J_{1}(\epsilon):=J(\epsilon)-J_{0}:=-\epsilon J_{2}(\epsilon), \\
J_{2}(\epsilon):=\int_{0}^{1} \frac{s^{p+1}\left(1-s^{p+1}\right)}{m(\epsilon, s) m(0, s)(m(\epsilon, s)+m(0, s))} d s .
\end{gathered}
$$

Now, we study the asymptotic behavior of $J_{2}(\epsilon)$ as $\epsilon \rightarrow 0$.

Lemma 4.1. (1) If $p>(3 q-1) / 2$, then $J_{2}(\epsilon) \rightarrow K_{2}$ as $\epsilon \rightarrow 0$.

(2) If $p<(3 q-1) / 2$, then as $\epsilon \rightarrow 0$,

$$
J_{2}(\epsilon)=K_{3}(1+o(1)) \epsilon^{(2 p-3 q+1) /(2(q+1))} .
$$

(3) If $p=(3 q-1) / 2$, then as $\epsilon \rightarrow 0$,

$$
J_{2}(\epsilon)=-\frac{1}{2(q+1)}(1+o(1)) \log \epsilon
$$

Proof. (1) Since $p>(3 q-1) / 2$, we have $(2 p-3 q-1) / 2>-1$. Therefore, by Lebesgue's convergence theorem, as $\epsilon \rightarrow 0$,

$$
J_{2}(\epsilon) \longrightarrow \frac{1}{2} \int_{0}^{1} \frac{s^{(2 p-3 q-1) / 2}\left(1-s^{p+1}\right)}{\left(1-s^{p-q}\right)^{3 / 2}} d s=K_{2}
$$

(2) We have the following two steps. 
Step 1. Assume that $p<(3 q-1) / 2$. We introduce $J_{3}(\epsilon)$ to approximate $J_{2}(\epsilon)$ :

$$
\begin{aligned}
J_{3}(\epsilon):= & \int_{0}^{1} \frac{s^{(2 p-q+1) / 2}}{\sqrt{s^{q+1}+\epsilon}\left(s^{(q+1) / 2}+\sqrt{s^{q+1}+\epsilon}\right)} d s \\
= & J_{4}(\epsilon, \delta)+J_{5}(\epsilon, \delta) \\
:= & \int_{0}^{\delta} \frac{s^{(2 p-q+1) / 2}}{\sqrt{s^{q+1}+\epsilon}\left(s^{(q+1) / 2}+\sqrt{s^{q+1}+\epsilon}\right)} d s \\
& +\int_{\delta}^{1} \frac{s^{(2 p-q+1) / 2}}{\sqrt{s^{q+1}+\epsilon}\left(s^{(q+1) / 2}+\sqrt{s^{q+1}+\epsilon}\right)} d s,
\end{aligned}
$$

where $0<\delta \ll 1$ is a fixed small constant. We study the asymptotic behaviors of $J_{3}, J_{4}$, and $J_{5}$ as $\epsilon \rightarrow 0$. Note that $0<(2 p-2 q+2) /(q+1)<1$ since $p<(3 q-1) / 2$. Then put $s=\epsilon^{1 /(q+1)} \tan ^{2 /(q+1)} \theta$ and $y=\tan (\theta / 2)$ to obtain

$$
\begin{aligned}
J_{3}(\epsilon)= & \frac{2}{q+1} \epsilon^{(2 p-3 q+1) /(2(q+1))} \int_{0}^{\tan ^{-1}(1 / \sqrt{\epsilon})} \frac{\tan ^{(2 p-2 q+2) /(q+1)} \theta}{1+\sin \theta} d \theta \\
= & \frac{2^{2(p+2) /(q+1)}}{q+1} \epsilon^{(2 p-3 q+1) /(2(q+1))} \\
& \times \int_{0}^{\tan (1 / 2)\left(\tan ^{-1}(1 / \sqrt{\epsilon})\right)} \frac{y^{(2 p-2 q+2) /(q+1)}}{(1+y)^{2(p+2) /(q+1)}(1-y)^{(2 p-2 q+2) /(q+1)}} d y \\
= & \frac{2^{2(p+2) /(q+1)}}{q+1}(1+o(1)) \epsilon^{(2 p-3 q+1) /(2(q+1))} \\
& \times \int_{0}^{1} \frac{y^{(2 p-2 q+2) /(q+1)}}{(1+y)^{2(p+2) /(q+1)}(1-y)^{(2 p-2 q+2) /(q+1)}} d y \\
= & K_{3}(1+o(1)) \epsilon^{(2 p-3 q+1) /(2(q+1))} .
\end{aligned}
$$

Similarly, we obtain

$$
\begin{aligned}
& J_{4}(\epsilon, \delta)=K_{3}(1+o(1)) \epsilon^{(2 p-3 q+1) /(2(q+1))}, \\
& J_{5}(\epsilon, \delta) \leq \frac{1}{\delta^{q+1}} .
\end{aligned}
$$

Since $p<(3 q-1) / 2$, this along with (4.10) implies that $J_{3}(\epsilon) / J_{4}(\epsilon, \delta) \rightarrow 1$ as $\epsilon \rightarrow$ 0 for a fixed $\delta$.

Step 2. We show that as $\epsilon \rightarrow 0$,

$$
\frac{J_{2}(\epsilon)}{J_{3}(\epsilon)} \longrightarrow 1
$$


Let an arbitrary $0<\delta \ll 1$ be fixed. Put

$$
\begin{aligned}
J_{2}(\epsilon)= & J_{6}(\epsilon, \delta)+J_{7}(\epsilon, \delta) \\
:= & \int_{0}^{\delta} \frac{s^{p+1}\left(1-s^{p+1}\right)}{m(\epsilon, s) m(0, s)(m(\epsilon, s)+m(0, s))} d s \\
& +\int_{\delta}^{1} \frac{s^{p+1}\left(1-s^{p+1}\right)}{m(\epsilon, s) m(0, s)(m(\epsilon, s)+m(0, s))} d s .
\end{aligned}
$$

Then for $0<\epsilon \ll 1$,

$$
\left|J_{7}(\epsilon, \delta)\right| \leq C_{2, \delta} \int_{\delta}^{1} \frac{1-s^{p+1}}{\left(1-s^{p-q}\right)^{3 / 2}} d s \leq C_{3, \delta} .
$$

Moreover, by (3.13), we obtain

$$
\begin{aligned}
& \left(1-\delta^{p+1}\right) \int_{0}^{\delta} \frac{s^{(2 p-q+1) / 2}}{\sqrt{s^{q+1}+\epsilon}\left(s^{(q+1) / 2}+\sqrt{s^{q+1}+\epsilon}\right)} d s \\
& \quad \leq J_{6}(\epsilon, \delta) \leq \frac{1}{\left(1-\delta^{p-q}\right)^{3 / 2}} \int_{0}^{\delta} \frac{s^{(2 p-q+1) / 2}}{\sqrt{s^{q+1}+\epsilon}\left(s^{(q+1) / 2}+\sqrt{s^{q+1}+\epsilon}\right)} d s .
\end{aligned}
$$

This implies

$$
\left(1-\delta^{p+1}\right) J_{4}(\epsilon, \delta) \leq J_{6}(\epsilon, \delta) \leq \frac{1}{\left(1-\delta^{p-q}\right)^{3 / 2}} J_{4}(\epsilon, \delta)
$$

By (4.11), (4.14), and (4.16), we see that $J_{7}(\epsilon, \delta)=o\left(J_{6}(\epsilon, \delta)\right)$ as $\epsilon \rightarrow 0$ for a fixed $\delta$ since $p<(3 q-1) / 2$. Then by (4.10), (4.11), (4.13), (4.14), and (4.16),

$$
\begin{aligned}
\left(1-\delta^{p+1}\right) & \leq \liminf _{\epsilon \rightarrow 0} \frac{J_{6}(\epsilon, \delta)}{J_{4}(\epsilon, \delta)} \\
& =\liminf _{\epsilon \rightarrow 0} \frac{J_{2}(\epsilon)}{J_{3}(\epsilon)} \leq \limsup _{\epsilon \rightarrow 0} \frac{J_{2}(\epsilon)}{J_{3}(\epsilon)} \\
& =\limsup _{\epsilon \rightarrow 0} \frac{J_{6}(\epsilon, \delta)}{J_{4}(\epsilon, \delta)} \leq \frac{1}{\left(1-\delta^{p-q}\right)^{3 / 2}} .
\end{aligned}
$$

By letting $\delta \rightarrow 0$, we obtain (4.12). Then by (4.10) and (4.12), we obtain (4.6).

(3) If $p=(3 q-1) / 2$, then by the asymptotic formula

$$
\tan ^{-1} x=\frac{\pi}{2}-\frac{1}{x}+O\left(\frac{1}{x^{3}}\right) \quad(x \gg 1)
$$


Taylor expansion of $\tan x$ at $x=\pi / 4$, and (4.10), we obtain

$$
\begin{aligned}
J_{3}(\epsilon) & =\frac{8}{q+1} \int_{0}^{\tan \left((1 / 2)\left(\tan ^{-1}(1 / \sqrt{\epsilon})\right)\right)} \frac{y}{(1+y)^{3}(1-y)} d y \\
& =\frac{1}{q+1} \int_{0}^{\tan \left((1 / 2)\left(\tan ^{-1}(1 / \sqrt{\epsilon})\right)\right)}\left\{\frac{1}{1-y}+\frac{1}{1+y}+\frac{2}{(1+y)^{2}}-\frac{4}{(1+y)^{3}}\right\} d y \\
& =\frac{1}{q+1}\left(-\log \left|1-\tan \left(\frac{1}{2} \tan ^{-1}\left(\frac{1}{\sqrt{\epsilon}}\right)\right)\right|+\log 2-\frac{1}{2}+o(1)\right) \\
& =\frac{1}{q+1}\left(-\log \left|\left(\frac{\pi}{2}-\tan ^{-1}\left(\frac{1}{\sqrt{\epsilon}}\right)\right)(1+o(1))\right|+\log 2-\frac{1}{2}+o(1)\right) \\
& =\frac{1}{q+1}\left(-\frac{1}{2}(1+o(1)) \log \epsilon+\log 2-\frac{1}{2}+o(1)\right) .
\end{aligned}
$$

By this and the same arguments as those in the proof of (2), we obtain (4.7).

\section{Proof of Theorem 2.1}

By summing up Lemmas 3.1, 3.2, 3.3, and 4.1, we now prove Theorem 2.1. By (2.15), (3.1), (4.1), and (4.5), we have

$$
\begin{aligned}
\mu(\lambda)^{(2 p-q+3) /(2(p-q))}= & \frac{\sqrt{2(q+1)}}{\gamma^{p+1}} \lambda^{(p+3) /(2(p-q))}\left\|w_{\lambda}\right\|_{\infty}^{(2 p-q+3) / 2} J(\epsilon(\lambda)) \\
= & \frac{\sqrt{2(q+1)}}{\gamma^{p+1}} \lambda^{(p+3) /(2(p-q))}\left(\frac{p+1}{q+1}\right)^{(2 p-q+3) /(2(p-q))} \\
& \times(1+\epsilon(\lambda))^{(2 p-q+3) /(2(p-q))}\left(J_{0}-\epsilon(\lambda) J_{2}(\epsilon(\lambda))\right) .
\end{aligned}
$$

Moreover, it is easy to check that

$$
\left(\frac{\sqrt{2(q+1)}}{\gamma^{p+1}}\right)^{2(p-q) /(2 p-q+3)} \frac{p+1}{q+1} J_{0}^{2(p-q) /(2 p-q+3)}=C_{1}
$$

By this, (5.1), and Taylor expansion, we obtain

$$
\begin{aligned}
\mu(\lambda)= & \left(\frac{\sqrt{2(q+1)}}{\gamma^{p+1}}\right)^{2(p-q) /(2 p-q+3)} \frac{p+1}{q+1} J_{0}^{2(p-q) /(2 p-q+3)} \lambda^{(p+3) /(2 p-q+3)} \\
& \times(1+\epsilon(\lambda))\left(1-\frac{2(p-q)}{(2 p-q+3) J_{0}} \epsilon(\lambda) J_{2}(\epsilon(\lambda))+o\left(\epsilon(\lambda) J_{2}(\epsilon(\lambda))\right)\right)
\end{aligned}
$$




$$
\begin{aligned}
= & C_{1} \lambda^{(p+3) /(2 p-q+3)} \\
& \times\left(1+\epsilon(\lambda)-\frac{2(p-q)}{(2 p-q+3) J_{0}}(1+o(1)) \epsilon(\lambda) J_{2}(\epsilon(\lambda))\right) .
\end{aligned}
$$

There are three cases to consider.

Case 5.1. Assume that $p>(3 q-1) / 2$. Then by Lemmas 3.3 and 4.1(1), we have

$$
\begin{aligned}
\mu(\lambda)= & C_{1} \lambda^{(p+3) /(2 p-q+3)}\left\{1+\left(1-\frac{2(p-q) K_{2}}{(2 p-q+3) J_{0}}\right) \epsilon(\lambda)+o(\epsilon(\lambda))\right\} \\
= & C_{1} \lambda^{(p+3) /(2 p-q+3)} \\
& \times\left\{1+\left(1-\frac{2(p-q) K_{2}}{(2 p-q+3) J_{0}}\right) K_{1}(1+o(1)) \lambda^{-2(p+1)(q+1) /((2 p-q+3)(q-1))}\right\} .
\end{aligned}
$$

This implies (2.6).

Case 5.2. Assume that $p<(3 q-1) / 2$. Then by Lemma 3.3, (4.6), and (5.3), we have

$$
\begin{aligned}
\mu(\lambda)= & C_{1} \lambda^{(p+3) /(2 p-q+3)} \\
& \times\left\{1-\frac{2(p-q)}{(2 p-q+3) J_{0}} K_{3} K_{1}^{(2 p-q+3) /(2(q+1))}(1+o(1)) \lambda^{-(p+1) /(q-1)}\right\} .
\end{aligned}
$$

This implies (2.8).

Case 5.3. Assume that $p=(3 q-1) / 2$. Then by Lemma 3.3, we have

$$
\log \epsilon(\lambda)=-\frac{2(p+1)(q+1)}{(q-1)(2 p-q+3)}(1+o(1)) \log \lambda
$$

This along with (4.7) and (5.3) implies

$$
\begin{aligned}
\mu(\lambda)= & C_{1} \lambda^{(p+3) /(2 p-q+3)} \\
& \times\left\{1-\frac{2(p-q)(p+1)}{(q-1)(2 p-q+3)^{2} J_{0}}\right. \\
& \left.\times K_{1}(1+o(1)) \lambda^{-2(p+1)(q+1) /((2 p-q+3)(q-1))} \log \lambda\right\} .
\end{aligned}
$$

This implies (2.10). Thus, the proof is complete. 
684 Two-parameter eigenvalue problems

\section{References}

[1] F. V. Atkinson, Multiparameter spectral theory, Bull. Amer. Math. Soc. 74 (1968), 127.

[2] P. Binding and P. J. Browne, Asymptotics of eigencurves for second order ordinary differential equations. I, J. Differential Equations 88 (1990), no. 1, 30-45.

[3] R. S. Cantrell, Multiparameter bifurcation problems for second order ordinary differential equations, Rocky Mountain J. Math. 12 (1982), no. 4, 795-806.

[4] M. Faierman, Two-Parameter Eigenvalue Problems in Ordinary Differential Equations, Pitman Research Notes in Mathematics Series, vol. 205, Longman Scientific \& Technical, Harlow, 1991.

[5] M. Faierman and G. F. Roach, Eigenfunction expansions associated with a multiparameter system of differential equations, Differential Integral Equations 2 (1989), no. $1,45-56$.

[6] T. Shibata, Asymptotic behavior of eigenvalues of two-parameter nonlinear SturmLiouville problems, J. Anal. Math. 66 (1995), 277-294.

[7] _ Two-parameter eigenvalue problems in nonlinear second order differential equations, Results Math. 31 (1997), no. 1-2, 136-147.

[8] - The effect of the variational framework on the spectral asymptotics for twoparameter nonlinear eigenvalue problems, Math. Nachr. 248/249 (2003), 168-184.

[9] B. D. Sleeman, The two parameter Sturm-Liouville problem for ordinary differential equations, Proc. Roy. Soc. Edinburgh Sect. A 69 (1971), 139-148.

[10] L. Turyn, Sturm-Liouville problems with several parameters, J. Differential Equations 38 (1980), no. 2, 239-259.

[11] H. Volkmer, On multiparameter theory, J. Math. Anal. Appl. 86 (1982), no. 1, 44-53.

Tetsutaro Shibata: The Division of Mathematical and Information Sciences, Faculty of Integrated Arts and Sciences, Hiroshima University, Higashi-Hiroshima 739-8521, Japan

E-mail address: shibata@mis.hiroshima-u.ac.jp 


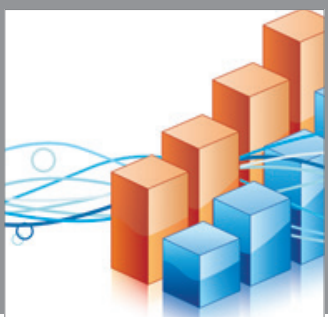

Advances in

Operations Research

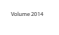

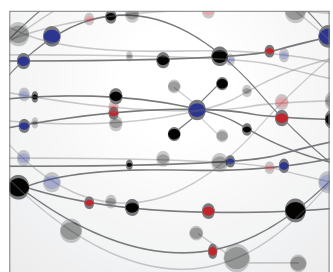

\section{The Scientific} World Journal
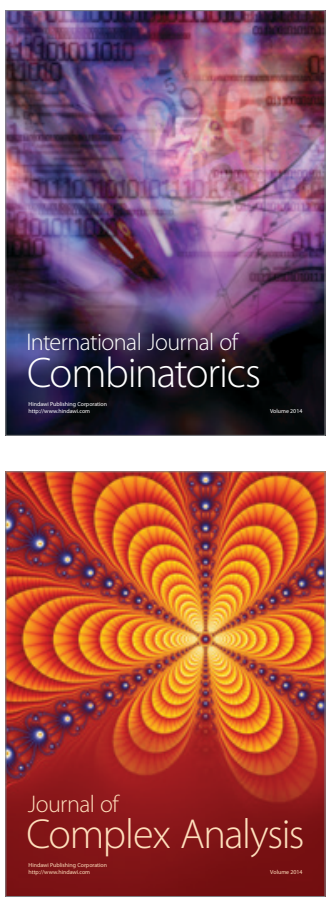

International Journal of

Mathematics and

Mathematical

Sciences
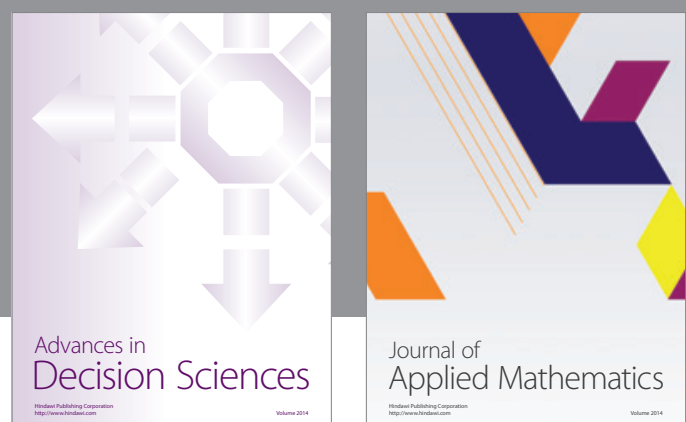

Journal of

Applied Mathematics
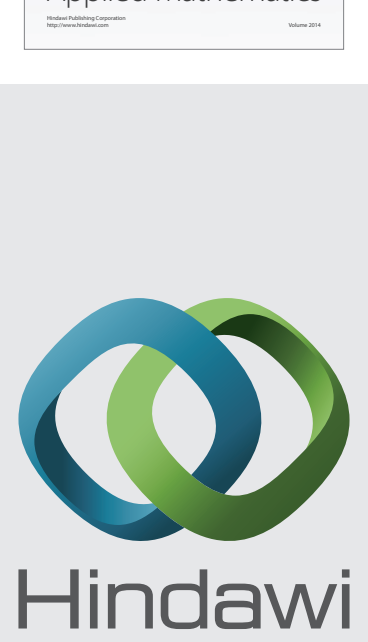

Submit your manuscripts at http://www.hindawi.com
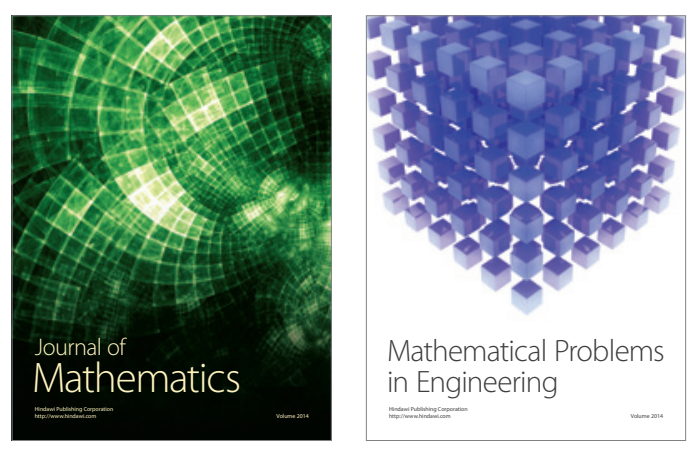

Mathematical Problems in Engineering
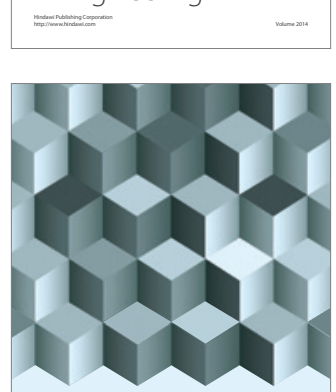

Journal of

Function Spaces
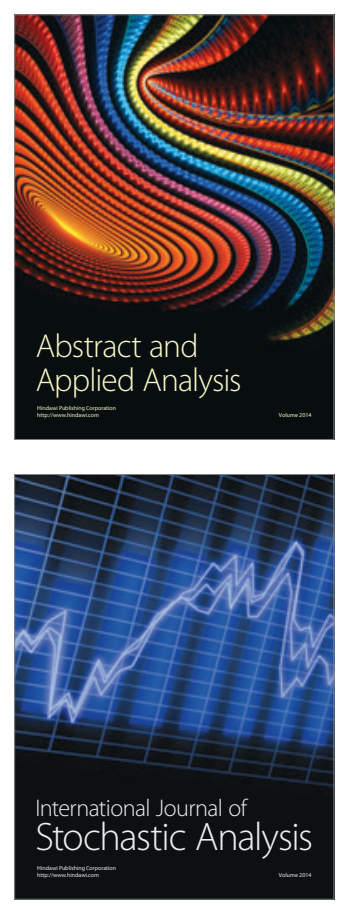

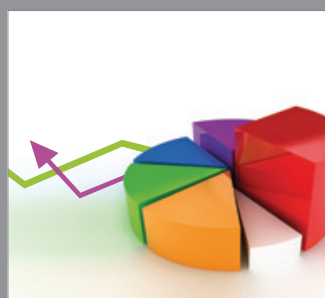

ournal of

Probability and Statistics

Promensencen
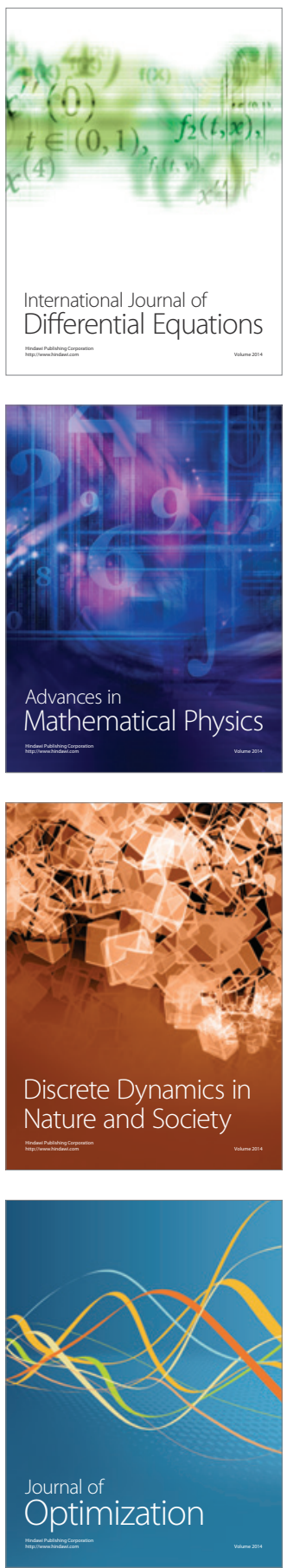